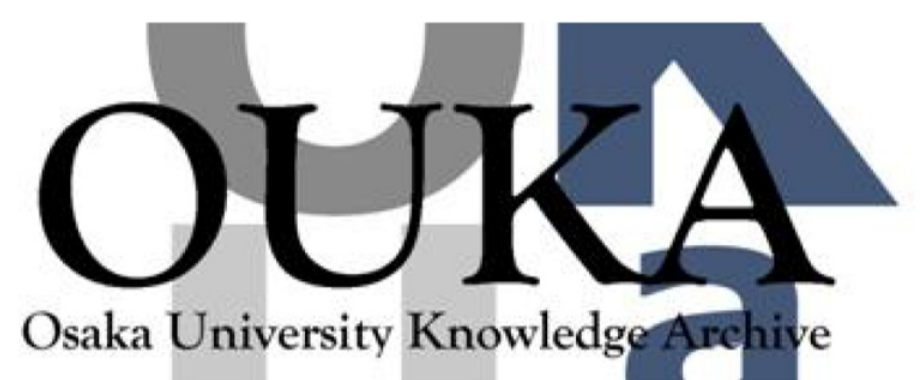

\begin{tabular}{|c|l|}
\hline Title & $\begin{array}{l}\text { Rotational viscosity in ferroelectric liquid } \\
\text { crystals estimated from transient light } \\
\text { scattering and dielectric properties }\end{array}$ \\
\hline Author(s) & Ozaki, M.; Hatai, T.; Nakao, K. et al. \\
\hline Citation & Journal of Applied Physics. 65(9) p. 3602-p. 3605 \\
\hline Issue Date & $1989-05-01$ \\
\hline oaire:version & VoR \\
\hline URL & https://hdl. handle.net/11094/75857 \\
\hline rights & \\
\hline Note & \\
\hline
\end{tabular}

Osaka University Knowledge Archive : OUKA

https://ir. Library. osaka-u. ac. jp/

Osaka University 


\section{Rotational viscosity in ferroelectric liquid crystals estimated from transient light scattering and dielectric properties}

Cite as: Journal of Applied Physics 65, 3602 (1989); https://doi.org/10.1063/1.342639

Submitted: 03 August 1988 . Accepted: 22 December 1988 . Published Online: 04 June 1998

M. Ozaki, T. Hatai, K. Nakao, and K. Yoshino
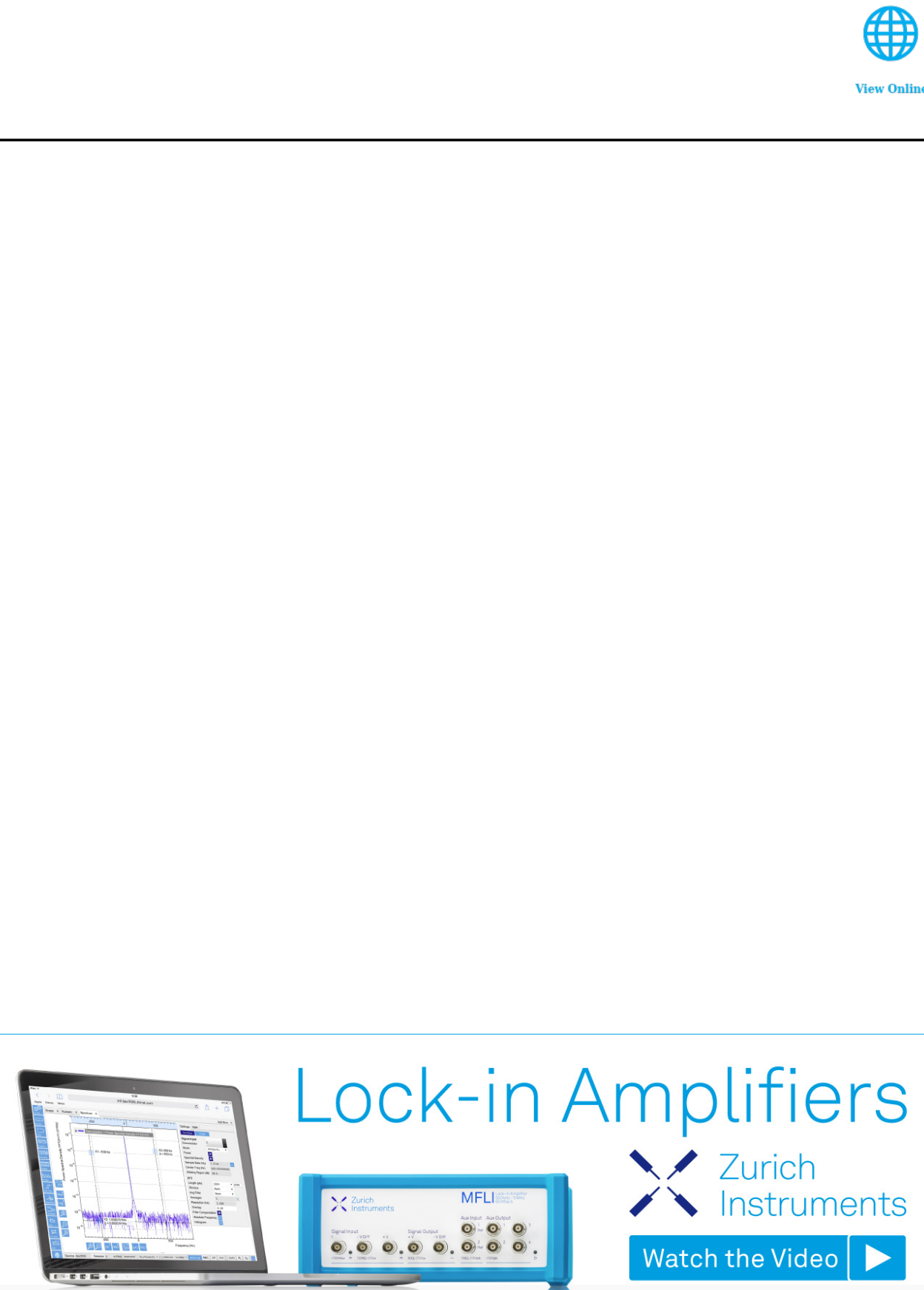


\title{
Rotational viscosity in ferroelectric liquid crystais estimated from transient light scattering and dielectric properties
}

\author{
M. Ozaki, T. Hatai, K. Nakao, and K. Yoshino \\ Department of Electronic Engineering, Faculty of Engineering, Osaka University, 2-1 Yamada-Oka, Suita, \\ Osaka, Japan
}

(Received 3 August 1988; accepted for publication 22 December 1988)

\begin{abstract}
A new method for the estimation of rotational viscosity of ferroelectric liquid crystals using transient light scattering (TSM) is reported. The relation between rotational viscosity, spontaneous polarization, and dielectric dispersion frequency is studied as a function of the racemization. The rotational viscosity is almost independent of spontaneous polarization and there is no obvious correlation between rotational viscosity and dispersion frequency. The dispersion frequency is confirmed to be inversely proportional to the square of the helical pitch. The rotational viscosity calculated from this relation is consistent with that from the new TSM method.
\end{abstract}

\section{NTRODUCTION}

Ferroelectric chiral smectic liquid crystals have attracted considerable attention from both fundamental and practical points of view, and a large number of studies have been carried out. ${ }^{1}$ Several types of electro-optical effects of ferroelectric liquid crystal have been reported, ${ }^{2-6}$ and their fast optical responses have stimulated the application to display devices. The optical response time $\tau$ of a ferroelectric liquid crystal under application of an electric field $E$ is related to the spontaneous polarization $P_{s}$ and the rotational viscosity $\eta$. In high field the simplest relationship without taking account of the switching mechanism ${ }^{4}$ is expected to be

$$
\tau=\eta / P_{s} E \text {. }
$$

To establish a high-respense speed of an optical cell with ferroelectric liquid crystal, therefore, it is important to prepare materials with large spontaneous polarization and low rotational viscosity.

In the earlier stage of the study of ferroelectric liquid crystal only a limited number of materials were used and their spontaneous polarizations were as small as the order of $10^{-9} \mathrm{C} / \mathrm{cm}^{2}$. Recently, however, a large number of ferroelectric liquid crystals have been synthesized and materials with large spontaneous polarizations have been reported. ${ }^{\text {-10 }}$ On the other hand, only a few measurements on $\eta$ were reported, and all of them were carried out using a very thin cell, surface-stabilized ferroelectric liquid crystal (SSFLC) cell. ${ }^{1 i-1.3}$

In a previous paper, transient light scattering at the instant of reversal of molecular alignment (domain switching) by polarity reversal of the applied electric field was reported and named TSM (transient scattering mode) by us. ${ }^{5}$ This scattering is easiiy obseryed as a decrease of transmission intensity through a cell that did not have polarizers or a special surface treatment. This response time is of the order of a microsecond in a material with a large spontaneous polarization. In this paper, we propose a new method to estimate the rotational viscosity using transient light scattering. In this method, optical measurement in a thicker cell $(>10$ $\mu \mathrm{m}$ ) compared with a SSFLC cell makes it easy to manufac- ture a sample cell in addition to no special surface treatment.

The racemization of optical isomers of the ferroelectric liquid crystal can remarkably change its characteristic properties such as helical pitch, spontaneous polarization, and dielectric constant. In the second half of this paper, we report the relationship among the rotational viscosity, the dispersion frequency of the dielectric constant, the spontaneous polarization, and the helical pitch by investigating the effect of the racemization.

\section{1\% EXPERIMENT}

Ferroelectric liquid crystals used in this study were DOBA-1-MPC ( $p$ decyloxybenzylidene- $p$-amino-1-methyipropylcinnamate $)^{14}$ and $3 \mathrm{M} 2 \mathrm{CPAOB}[(2 S, 3 S)-3$-methyl-2-chloropentanoic acid 4',4"-alkyloxy-biphenyl ester . $^{715}$ Details of preparation of these liquid crystals were previousiy reported by us. The maximum magnitudes of spontaneous polarization of DOBA-1-MPC and 3M2CPAOB in the Sm-C* phase are about 20 and 200 $\mathrm{nC} / \mathrm{cm}^{2}$, respectively. The sample was sandwiched between ITO (In-Sn oxide) coated glass plates with PET (polyethyleneterephthalate) film as a spacer.

The sample cell was set in the optical path of a He-Ne laser $(632.8 \mathrm{~nm})$ without polarizers, and the change of transmission intensity by stepwise polarity reversal of electric field was monitored by a photodiode and stored by a digital memory. The polarization current accompanied by domain switching was also measured simultaneously from the voltage change over a series resistor $(327 \Omega$ or $4.7 \mathrm{k} \Omega$ ).

The spontaneous polarization was calculated from the area under the polarization current curve. The dielectric constant was measured by evaluating that part of the current whose phase was shifted by $90^{\circ}$ from that of the applied voltage with a lock-in amplifier (PAR, 5204). ${ }^{16}$ The applied voltage was sufficiently low not to perturb the molecular alignment.

The pitch of the helicoidal structure was evaluated by the diffraction pattern of a $\mathrm{He}-\mathrm{Ne}$ laser with a sufficiently thick cell $(>350 \mu \mathrm{m})$ in order to avoid surface effects. 


\section{IR. RESULTS ANO DISCUSSION}

\section{A. Estimation of the rotational viscosity from transient} light scattering

Figures $1(a)$ and 1 (b) show typical waveforms of transmitted light through a cell and the simultaneously measired polarization reversal current when the polarity of applied voltage was reversed stepwise in the $25 \% \mathrm{~m}$ cell of $3 M 2 C P O O B$ (the length of alkoxychain $n=8$ ). At the in:stance of polarization reversal, the He-Ne laser light through the cell is scattered extensively, resulting in a rapid decrease of transmission intensity.

The response time of the decrease of transmission intensity becomes shorter with increasing applied voltage. Figure 2 shows field dependencies of the response time in $3 \mathrm{M} 2 \mathrm{CPOOB}$ at various temperatures. In this study, the response time $\tau$ of the transmission change is defined as the time taken for switching from $10 \%$ to $90 \%$ of the maximum transmission change. As is evident from Fig. 2, the response time is decreasing with increasing applied feld. In higher electric fields, $\tau$ is proportional to the inverse of the electric field as expected from Eq. (1).

The result shown in Fig. 2 agrees well with relationship (1). Therefore, one can evaluate $\eta$ from the slope $\eta / P_{s}$ of this figure and $P_{s}$ calculated from the polarization current. Figure 3 shows temperature dependences of $\eta$ in $3 \mathrm{M} 2 \mathrm{CPHPOB}(n=7), 3 \mathrm{M} 2 \mathrm{CPOOR}(n=8)$, and $3 \mathrm{M} 2 \mathrm{CPNOB}(n=9)$. In all cases, $\eta$ increases with decreasing temperature. However, it is almost independent of the length of the alkoxychain, which will be discussed below.

Several trials of the estimation of $\eta$ have previously been reported using SSFLC cells, in which the time required for an optical transmission changes from $10 \%$ to $90 \%,{ }^{12}$ or the time between the polarization current peak and the polarity reversal ${ }^{11,13}$ were used as the characteristic time, $\tau$. Recently, a new method for the evaluation of $\eta$ has been reported by Dahl, Lagerwall, and Skarp ${ }^{17}$ which is based on a simple model taking an elastic torque but not a switching mechanism into consideration. In this method, $\eta, P_{s}$, and the elastic constant were estimated from the width at half-maximum

(a)

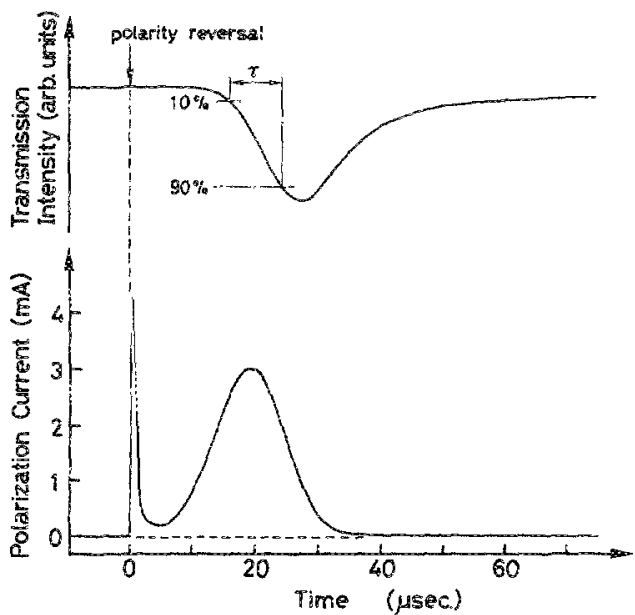

FIG. 1. Typical waveforms of transmission change (a) and polarization current $(b)$, at the instance of polarity reversal of applied field $(28 \mathrm{kV} / \mathrm{cm})$ in a $25-\mu \mathrm{m}$ cell of $3 \mathrm{M} 2 \mathrm{CPOOB}$ at $51.5^{\circ} \mathrm{C}$.

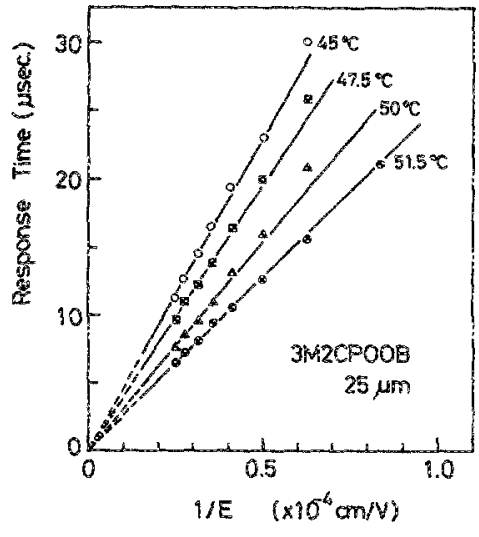

FIG. 2. Dependencies of the response time of TSM on applied electric fidd in $25-\mu \mathrm{m}$ cell of $3 \mathrm{M} 2 \mathrm{CPOOR}$ at various termperatures.

of the polarization current peak, the height of the current peak, and the time between the current peak and polarity reversal. A similar method which uses half-maximum of polarization current peak and a simple model taking account of the contribution of dielectric anisotropy instead of elastic torque, has been also reported. ${ }^{18}$

Viscosities of our samples from Dahl's method are also shown in Fig. 3 for reference. As is evident from this figure, calculated values by the two methods almost agree. Al though the details of the switching mechanism of TSM are not clear at this stage, the response time is found to be useful as the characterizing time in estimating rotational viscosity with expression (1).

\section{B. Racemization eftect of rotational viscosity}

From the consideration of symmetry for the appearance of ferroelectricity in liquid crystal, the spontaneous polarization is required to be perpendicular to both the smectic layer normal and also the molecular long axis. In ferroelectric liquid crystals, therefore, two directions of spontaneous polatization are possible. ${ }^{19}$ When the layer normal, director, and spontaneous polarizations form a right-handed coordinate system, the direction of $P_{s}$ is defined as positive, $P_{s}(+)$. If they form a left-handed one, the direction of $P_{s}$ is defined as negative, $P_{s}(-)$. These directions are intrinsic to the nature of a specific material except for a few special cases. ${ }^{20}$ It should also be pointed out that two optical isomers with

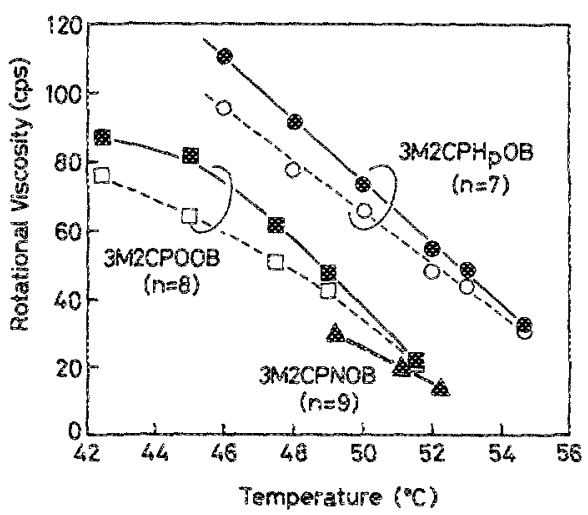

FIG. 3. Temperature dependercies of rotational viscosity of $3 M 2 \mathrm{CPAOB}$ (alkoxychain iength $n=7-9$ ) abtained from the response time of TSM by relation (1) (solid line) and also from Dah's method (dashed line). 
opposite absolute configuration ( $S$ or $R$ ) are of opposite spontaneous polarization direction. (R)-DOBA-1-MPC has a negative spontaneous polarization direction, $P_{s}(-)$, while $(S)$-DOBA-1-MPC has an opposite one. Therefore, racemization of these isomers decreases the magnitude of spontaneous polarization as shown in Fig. 4.

Figure 4 also shows response time of TSM and $\eta$ estimated from this as a function of concentration of $(S)$ DOBA-1-MPC in (R)-DOBA-1-MPC. It should be noted that $\eta$ does not depend strongly on the concentration of $(S)$ DOBA-1-MPC as compared to the remarkable decrease of spontaneous polarization. According to expression (1), the response time $\tau$ is related to $\eta$ and $P_{s}$ under a constant applied field. Therefore, Fig. 4 implies that the increase of the response time is due to the decrease of $P_{s}$. In other words, the rotational viscosity does not depend on the concentration of an optical impurity, that is, on the magnitude of spontaneous polarization.

\section{Rotational viscosity and dielectric dispersion frequency}

When the field is exerted parallel to a smectic layer, there exist two dielectric relaxations. ${ }^{21}$ One is due to the contribution of the motion of dipole moments associated with the fluctuation of the azimuthal angle of a long molecular axis around a smectic layer normal (Goldstone mode). The other originates from the contribution of the soft mode corresponding to fuctuation of the tilt angle.

Recently we have pointed out that the contribution of the soft mode to the dielectric constant of a ferroelectric liquid crystal with a large spontaneous polarization is remarkable in the vicinity of the phase transition temperature $T_{c}$ between $\mathrm{Sm}-A$ and $\mathrm{Sm}-C^{*}$ phases. ${ }^{22}$ In the temperature range studied here, however, the contribution of the Goldstone mode should be dominant.

The dispersion frequency of the Goldstone mode, in general, is relatively low, of the order of several tens $\mathrm{Hz}$ to several $\mathrm{kHz}$. However, this frequency indicates a remarkable

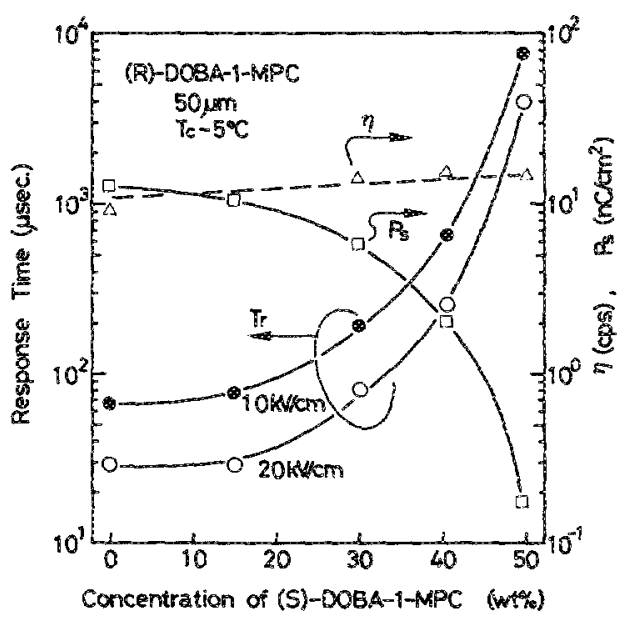

FIG. 4. The response time of TSM, the spontaneous polarization and the rotational viscosity as a function of weight concentration of $(S) \cdot D O B A-1$ $\mathrm{MPC}$ in $(R)$-DOBA-1-MPC. dependence on the molecular structure of a liquid crystal. ${ }^{23}$ In a previous paper ${ }^{15}$ we have reported that the dispersion frequency of dielectric constant is markedly dependent on the length $n$ of an alkoxy chain in 3M2CPAOB $(n=7-9)$. In $3 \mathrm{M} 2 \mathrm{CPOOB}(n=8)$, dispersion frequency is about several tens of $\mathrm{Hz}$, while it is around $800 \mathrm{~Hz}$ and $1-3 \mathrm{kHz}$ in $3 \mathrm{M} 2 \mathrm{CPNOB}(n=9)$ and $3 \mathrm{M} 2 \mathrm{CPH} \mathrm{OB}(n=7)$, respectively.

To investigate the relation between the rotational viscosity $\eta$ and the dispersion frequency $f$ in these materials, transient light scattering and polarization current were measured and $\eta$ was evaluated. Figure 3 shows temperature dependencies of $\eta$ in 3M2CPAOB $(n=7-9)$. It should be noted that $\eta$ is almost independent of $n$, and there is not an obvious correlation between $\eta$ and $n$ such as an odd-even effect corresponding to the relation between $n$ and $f$. Therefore, there is no correlation between rotational viscosity and dispersion frequency of the dielectric constant.

According to a simple free-energy expansion neglecting the fiexoelectric coupling between polarization and molecular tilt, the relaxation frequency of the Goldstone mode $\left(f_{G}\right)$ is expressed as follow ${ }^{24}$;

$$
f_{G}=K q^{2} / 2 \pi \eta,
$$

where $K$ is the elastic constant and $q$ is the wave vector of the helicoidal structure, that is, $q=2 \pi / p$, where $p$ is the helical pitch. In a series of $3 \mathrm{M} 2 \mathrm{CPAOB}(n=7-9)$, the intrinsic helical pitch is not significantly infuenced by alkoxy chain length. From this expression, therefore, it is suggested that a large variation of the dispersion frequency is caused by a difference of the elastic constant $\mathbb{K}$.

\section{Dispersion trequency and helical pitch}

Optical isomers with opposite absolute configurations are of opposite helical twist sense as well as spontaneous polarization direction, and the racemization of them extends the pitch of the helicoidal structure $p$ as shown in Fig.5. In order to investigate the relation between $p$ and $f, f$ of DOBA1-MPC was also measured as a function of the concentration of the optical isomer, which was shown in the same figure.

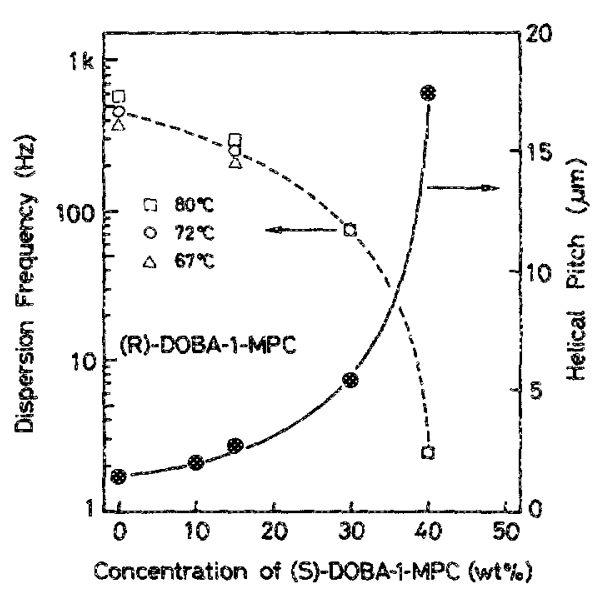

FIG. 5. The dispersion frequency of the dielectric constant and helical pitch as a function of the weight concentration of $(S)$-DOBA-1-MPC in $(R)$ DOBA-1-MPC. 


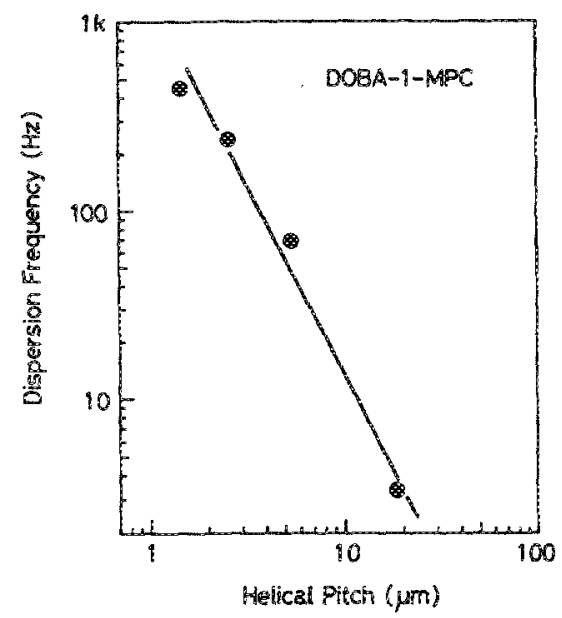

FIG. 6. The dispersion frequency of the dielectric constant as a function of the helical pitch for various mixtures of $(S)-D O B A-1-M P C$ and $(R)$ DOBA-1-MPC.

The dispersion frequency became low upon increasing the concentration of optical impurity.

As mentioned above, from expression $(2), f$ is predicted to be inversely proportional to $p^{2}$. To verify this relation, $f$ was plotted in Fig. 6 as a function of $p$ which was controlled by the concentration of the optical impurity. As is evident from this figure, the slope is about -2 , and the relation $f \propto 1 / p^{2}$ is verified.

According to expression (2), if it is assumed that the elastic constant $K$ is constant, the slope of a plot of $f$ us $p^{-2}$ gives $K / \eta$ and $\eta$ is calculated from it. One can obtain $14-48$ $C^{P}$ for $y$ using for the elastic constant $a$ of $3 \times 10^{-12}-10^{-11}$ N. This value is consistent with the one obtained from transient light scattering shown in Fig. 4. However, this calculation contains an assumption. That is, it is assumed that the elastic constant is constant in spite of a remarkable change of helical pitch. In fact, as shown in Fig. 6, points plotted in the logarithmic graph are not exactly on the straight line. This may suggest that $K$ depends on the concentration of optical impurity.

\section{CONCLUSION}

In this study, a new method for the estimation of rotational viscosity of ferroelectric liquid crystal using transient light scattering (TSM) was proposed. It was empirically found that the response time of TSM was useful as the characterizing time in estimating rotational viscosity with the relation $\tau=\eta / P_{s} E$. The rotational viscosity estimated from this method was almost independent of spontaneous polarization, and there was no obvious correlation between rotational viscosity and dielectric dispersion frequency.

From the investigation of the racemization, it was verified that dispersion frequency is inversely proportional to the square of the helical pitch $\left(f=2 \pi K / p^{2} \eta\right)$. The rotational viscosity calculated from this expression is consistent with that from the response time of TSM.

${ }^{1}$ R. B.Meyer, L. Liebert, L. Strzelecki, and P. Keller, J. Fhys.(Paris) 36, L69 (1975).

${ }^{2}$ K. Yoshino, K. G. Balakrishnan, T. Uemoto, and Y. Inuishi, Jpn. J. Appl. Phys. 17, 597 (1978).

${ }^{3}$ Y. Iwasaki, K. Yoshino, T. Uemoto, and Y. Inuishi, Jpn. J. Appl, Phys 18, 2323 (1979).

${ }^{4}$ N. A. Clark and S. T. Lagerwall, Appl. Phys. Lett. 36, 899 (1980).

${ }^{5} \mathrm{~K}$. Yoshino and M. Ozaki, Ferroelectrics \$9, 145 (1984).

"M. Ozaki, S. Kishio, K. Yoshino, N. Mikami, R. Higuchi, and T. Sakurai, Jpn. I. Appl. Phys. 26, Suppl. 26-2, 110 (1987)

TT. Sakurai, N. Mikami, R. Higuchi, M. Honma, M. Ozaki, and K. Yoshino, J. Chem. Soc. Chern. Commun., 978 (1986).

${ }^{8} \mathrm{~K}$. Yoshino, M. Ozaki, H. Taniguchi, M. Ito, K. Satoh, N. Yamasaki, and T. Kilazume, Ipn. J. Appl. Phys. 26, L77 (1987).

${ }^{9} \mathrm{H}$. Taniguch, M. Ozaki, K. Yoshino, K. Satoh, and N. Yamasaki, Ferroelectrics 77, 137 (1988).

${ }^{10}$ K. Mohr, S. Kohler, K. Worm, G. Pelal, S. Diele, H. Zaschke, D. Demus, G. Andersson, B. Dahl, S. T. Lagerwall, K. Skarp, and N. Stebler, Mol. Cryst. Liq. Cryst. 146, 151 (1987).

${ }^{11}$ K. Skarp, I. Dahl, S. T. Lagerwall, and B. Stebler, Mol. Cryst. Liq. Cryst. $114,283(1384)$.

12K. Flatischler, K. Skarp, S. T. Lagerwall, and B. Stebler, Moi. Cryst. Liq. Cryst. 131, 21 (1985).

${ }^{13}$ S. S. Bawa, A. M. Biradar, and S. Chandra, Jpn. I. Appl. Phys. 26, 189 (1987)

${ }^{14}$ T. Sakurai, K. Sakamoto, M. Honma, K. Yoshino, and M. Ozaki, Ferroelectrics $58,21(1984)$.

${ }^{15}$ M. Ozaki, K. Yoshino, T. Sakurai, N. Mikami, and R. Higuehi, $y$. Chem. Phys. 86, 3648 (1987).

:D. Meyerhofer, $\overline{3}$.Appl.Phys 46,5084 (1975).

17. Dahl, S. T. Lagerwall, and K. Skarp, Phys. Rev. A 36, 4380 (1987).

${ }^{15} \mathrm{~S}$. Kimura, S. Nishiyana, Y. Ouchi, H. Takezoe, and A. Fukuda, Jpn. J. Appl. Phys. 26, L255 (1987)

${ }^{19}$ N. A. Clark and S. T, Lagerwall, Ferroelectrics 59, 25 (1984).

${ }^{20}$ N. Mikami, R. Higuchi, T. Sakurai, M. Ozaki, and K. Yoshino, Jpn. J. Appl. Phys. 25, L833 (1986).

${ }^{2}$ R. Bline and B. Zeks, Phys. Rev. A 18, 740 (1978).

${ }^{22} \mathrm{~K}$. Yoshimo, K. Nakao, H. Taniguchi, and M. Ozaki, J. Phys. Soc. Ipn. 56, $4150(1987)$

${ }^{23}$ K. Yoshino, M. Ozaki, T. Sakurai, K. Sakamoto, and M. Honma, Jpn. J. Appl. Phys. 23, L175 (1984).

${ }^{24} \mathrm{G}$. Durand snd Ph. Martinot-Lagarde, Ferroelectrics 24,89 (1980). 